La notion de neutralité de l'État (séparation Église/État) dans le discours politique irlandais en matière d'éducation : entre confusion sémantique et stratégie d'évitement, années 1970-2000

\title{
Karin Fischer
}

\section{(2) OpenEdition \\ Journals}

\section{Édition électronique}

URL : http://journals.openedition.org/etudesirlandaises/4541

DOI : 10.4000/etudesirlandaises. 4541

ISSN : 2259-8863

\section{Éditeur}

Presses universitaires de Rennes

Édition imprimée

Date de publication : 30 juin 2015

Pagination : 149-164

ISBN : 978-2-7535-4082-8

ISSN : 0183-973X

\section{Référence électronique}

Karin Fischer, «La notion de neutralité de l'État (séparation Église/État) dans le discours politique irlandais en matière d'éducation : entre confusion sémantique et stratégie d'évitement, années 1970-2000 », Études irlandaises [En ligne], 40-1 | 2015, mis en ligne le 30 juin 2017, consulté le 01 mai 2019. URL : http://journals.openedition.org/etudesirlandaises/4541 ; DOI : 10.4000/ etudesirlandaises.4541 


\title{
La notion de neutralité de l'État (séparation Église/État) dans le discours politique irlandais en matière d'éducation : entre confusion sémantique et stratégie d'évitement, années 1970-2000
}

\author{
Karin FISCHER
}

Université d'Orléans

\section{Résumé}

L'article se penche sur les évolutions et permanences dans l'emploi et la définition implicite en contexte de la notion de neutralité de l'État vis-à-vis des Églises sur la période 1970-2011 en République d'Irlande (avec une attention particulière au domaine de l'éducation). Une analyse diachronique et synchronique des discours politiques irlandais autour de cette question aide à mieux comprendre le caractère persistant de "l'illusion de neutralité " mise en évidence par Bill Kissane notamment. À partir d'une conception idiosyncratique et problématique de la neutralité de l'État en matière de religion largement partagée jusqu'aux années 1970, on reconnaît par la suite des réponses politiques diverses, mais correspondant le plus souvent à des stratégies d'évitement ou de contournement de la question du sens et de l'application de cette notion de neutralité d'un point de vue institutionnel en Irlande. En marge de ce courant toujours dominant, des voix, plus nombreuses mais encore rares, se font entendre en faveur d'une séparation complète de l'Église et de l'État et de l'élimination concomitante de toute forme de discrimination religieuse en République d'Irlande.

Mots clés : éducation, Église catholique d'Irlande, société et religion, débat et sphère publics, État (République d'Irlande), neutrality

\section{Abstract}

With a view to better understanding why the "illusion of neutrality" in the Republic of Ireland as identified notably by Bill Kissane has persisted up to now, this article offers an analysis of political discourse on state neutrality and the notion of secularity over the 1970-2011 period, with a particular focus on the area of education policy. While a problematic and rather idiosyncratic conception of the relationship between Church and State remained dominant up to the 1970s, a comparative analysis of statements across the political scene since that time shows that most Irish politicians have developed strategies to avoid confronting the issue of state neutrality, its meaning and the gap between theory and practice (implications on Irish institutions) head on. Calls for a complete separation of Church and State and the elimination of all forms of religious discrimination in the Republic of Ireland, while more numerous and perhaps more vocal than in the past, have remained comparatively rare, and mostly outside the Irish political mainstream.

Keywords: education, Irish Catholic Church, society and religion, public debates, Irish State (Republic of Ireland), neutrality 
À partir d'une étude de discours politiques (débats parlementaires, productions gouvernementales, prises de position dans les médias), le présent article se propose de mettre en évidence les principales variations, évolutions ou permanences dans l'emploi et la définition implicite en contexte de la notion de neutralité de l'État au cours des trente dernières années, avec une attention particulière au domaine de l'éducation. Le point de départ est une recherche sur les usages de l'expression "state neutrality» et ce qu'elle a pu signifier dans la bouche d'hommes politiques successifs. Cette notion est étroitement liée à la question de la séparation de l'Église et de l'État, d'où l'intérêt d'explorer parallèlement le sens donné au terme "secular» (et à ses dérivés) et l'usage qui en est fait.

Le rapport complexe entretenu par l'État et la société irlandaise avec l'idée de séparation de l'Église et de l'État a déjà été abordé par différents auteurs, à commencer par Desmond M. Clarke dans son ouvrage précurseur Church and State in Modern Ireland', et plus récemment par des spécialistes du droit constitutionnel irlandais tels que Gerry Whyte ${ }^{2}$ ou Eoin Daly ${ }^{3}$, ou encore des politologues comme Bill Kissane dans un article dont le titre annonce clairement le propos, "The illusion of state neutrality in a secularising Ireland ${ }^{4} »$. Ces auteurs ont mis en évidence le fossé entre l'apparence de neutralité dans la Constitution irlandaise (du moins depuis l'amendement de 1972 qui a mis fin sur le papier à la " position privilégiée " de l'Église catholique en tant que religion de la majorité de la population irlandaise) et la réalité du système institutionnel irlandais, un fossé permis jusqu’à présent par l'ambivalence d'une Constitution se situant de ce point de vue sur une "ligne de faille idéologique " (d'après l'expression de Gerry Whyte) ${ }^{5}$, mais aussi facilité selon Eoin Daly et d'autres par des choix interprétatifs problématiques. Étant entendu qu'aucun État jusqu'à présent ne peut se prévaloir d'une complète neutralité de l'État vis-à-vis des intérêts religieux ou/et privés dans l'éducation, que celle-ci se présente ou se conçoive sous l'appellation de laïcité ou non (ÉtatsUnis, France, Italie, Turquie etc.), l'Irlande reste néanmoins un cas particulier de

1. Desmond M. Clarke, Church and State in Modern Ireland, Cork, Cork University Press, 1985.

2. Gerry Whyte, "Religion and Education - The Irish Constitution ", communication au colloque organisé par Trinity College Dublin et la Irish Human Rights Commission sur le thème «Religion and Education, A Human Rights Perspective ", 27 nov. 2010; Gerry Whyte, "Religion and the Irish Constitution ", John Marshall Law Review, 725, 1997.

3. Eoin Daly, Religion, Law and the Irish State, Dublin, Clarus Press, 2012.

4. Bill Kissane, "The Illusion of state neutrality in a secularising Ireland ", West European Politics, vol. 26, 2003 (numéro "Church and State in Contemporary Europe "), p. 73-94.

5. Contrairement à la Constitution essentiellement lä̈que de 1922, la Constitution de 1937 contient un mélange conflictuel entre l'influence de la pensée libérale du $19^{\mathrm{e}}$ siècle et l'idéologie catholique en matière de droits, de démocratie, de religion, de partage des rôles dans la société et d'éducation. Paul Brennan, «La Délaïcisation de l'État (1922-1937) ", in Paul Brennan, La Sécularisation en Irlande, Caen, Presses Universitaires de Caen, 1998, p. 119-120. 
ce point de vue étant donné une participation institutionnelle plus forte qu'ailleurs de la religion dans les domaines éducatif, de santé, et social.

Il ne s'agira pas ici de revenir sur ce qui peut être considéré comme un état de fait, sur la base du travail de ces auteurs, mais de chercher à comprendre le rapport que la classe politique irlandaise et ses principaux partis ont pu entretenir avec cette "illusion " de neutralité. Un coup d'oeil superficiel aux débats parlementaires touchant à cette question depuis 1972 semble indiquer un consensus quasi-universel sur le sujet de la séparation de l'Église et de l'État, tous les discours indiquant un positionnement favorable, mais également le plus souvent l'idée que celle-ci est déjà une réalité en République d'Irlande ${ }^{6}$. Pourtant des universitaires en nombre croissant pointent du doigt les contradictions persistantes, et la question refait surface régulièrement malgré tout sur la scène publique. Comment ce hiatus a-t-il donc pu être perpétué? Les discours politiques sur le sujet sont-ils révélateurs d'une confusion générale ou bien de positionnements délibérés visant à entretenir l'illusion dont parle Bill Kissane? Y a-t-il eu une évolution au cours des trente dernières années? Peut-on parler d'une mentalité politique spécifique de ce point de vue? Peut-on identifier des oppositions politiques de fond au-delà du consensus apparent?

Dans l'espace limité d'un article, il est difficile de rendre compte précisément des différentes tendances politiques et de s'attaquer de manière détaillée aux questions de définition liées à des termes comme "secular " par exemple. Nous nous contenterons donc de proposer une vision d'ensemble à partir d'exemples choisis pour leur représentativité ou leur caractère révélateur. Après un premier aperçu général fondé sur quelques exemples d'usages de "secular " sur la scène politique et médiatique irlandaise, nous chercherons à rendre compte, de manière toujours synthétique, d'une certaine évolution chronologique, et explorerons pour finir la possibilité d'une typologie politique à partir de l'analyse de discours.

\section{Vicissitudes du mot " secular » et de ses dérivés en République d'Irlande}

L'analyse du terme "secular» et de ses usages est compliquée ici par la question de la traduction, mais aussi par l'idée avancée par certains que ce terme aurait un sens spécifique en Irlande. C'était en tout cas l'argument employé par Richard Clarke, prélat de l'Église d'Irlande, qui affirmait lors d'un entretien en 2011 que le terme avait un sens différent en Irlande et sur le continent et qu'en raison de cet

6. Par exemple déclaration du Premier Ministre Bertie Ahern (Fianna Fáil) : "In the great tradition of Daniel O'Connell, we have proper separation of church and State and we avoid confessional politics ", 13 décembre 2005, Dáil Éireann Debates, vol. 612, nº 105. 
emploi spécifique, il n'était pas possible de comparer la situation en Irlande et en Italie par exemple 7 .

De fait, l'usage le plus commun fait de « secular " dans les cercles politiques et médiatiques irlandais à la fin du $\mathrm{Xx}^{\mathrm{e}}$ siècle l'éloigne nettement de la définition généralement admise du terme comme de son sens historique. Dans le contexte médiatique, au fil des articles publiés dans les années 1990 et 2000, l'adjectif «secular» sonnait parfois presque comme un gros mot, l'amalgame fréquent avec le terme "secularist " contribuant à un glissement sémantique vers le sens d'" anti-religieux " ou au mieux d'" irreligieux ", avec le sous-entendu ou présupposé supplémentaire chez certains qu' "irreligieux " ou non religieux signifierait nécessairement "immoral ${ }^{8}$ ». Cette tendance (plus souvent contredite dans les médias irlandais depuis quelques années) contrastait avec l'usage plus neutre et plus proche de son acception générale que l'on retrouvait au $\mathrm{XIX}^{\mathrm{e}}$ siècle, lorsque les écoles primaires nationales irlandaises ("National Schools») par exemple étaient censées faire la distinction entre instruction profane/laïque/non religieuse et instruction religieuse (distinction souvent marquée par l'usage de petits panneaux indiquant "secular instruction" ou " religious instruction " affichés dans la salle de classe en fonction du sujet de la leçon) ${ }^{9}$.

Le glissement sémantique fréquent dans l'Irlande de la fin du $\mathrm{xx}^{\mathrm{e}}$ siècle semble être essentiellement fondé sur une interprétation problématique du concept de "secularism", qui, dans un amalgame avec le concept sociologique de "secularisation ", peut induire une volonté d'écarter la religion de la vie sociale d'un pays (jusqu'à la doctrine du rejet complet de la religion), une volonté qui s'éloigne en réalité de la notion de séparation institutionnelle des Églises et de l'État à laquelle le terme «secularism» fait plus généralement référence. Ce type d'amalgame ou de confusion sémantique peut être illustré également par le rejet d'institutions laïques comme étant de nature dictatoriale et anti-religieuse et la confusion notamment avec le régime d'athéisme officiel de l'URSS : lors d'un débat parlementaire en 1987 face aux députés Proinsias de Rossa et Michael D. Higgins, Patrick Cooney du Fine Gael faisait ainsi un parallèle entre l'idée d'un système éducatif public

7. Entretien avec Richard Clarke, Dublin, juin 2011.

8. Parmi les commentateurs les plus véhéments sur le sujet dans les médias (contredits de plus en plus souvent dans d'autres articles ou les lettres au journal), on peut citer John Waters et Breda O'Brien (cette dernière affiliée au Iona Institute, une organisation catholique). Par exemple, John Waters, "Attack on religious instruction in schools is wrong ", Irish Times, 13 avril 2012; Breda O’Brien, "Passing flawed legislation on abortion would confirm groupthink of Irish elite ", Irish Times, 26 mai 2013. Dans l'Irish Independent, sur les « sécularistes agressifs ", "Don't scapegoat Catholic Church for the planners' own failures ", Irish Independent, 7 septembre 2007; Eric Conway (letter to the editor), "Narrow minds and Catholics ", Irish Independent, 21 juin 2007. Également Ruth Dudley Edwards, "God help us all in the face of the militant secularists ", Irish Independent, 25 mai 2008.

9. Tony Lyons, "Thomas Wyse and non-denominational education in Ireland, 1830-1845 ", dans Deirdre Raftery et Karin Fischer (dir.), Educating Ireland - Schooling and Social Change, 1700-2000, Dublin, Irish Academic Press, 2014, p. 55-56. 
"laïque " (secular) et la Russie communiste ${ }^{10}$. Une telle confusion peut paraître d'autant plus surprenante que ce sont au contraire les États-Unis qui représentent l'un des modèles les plus connus d'État laïque, avec une séparation État-Églises particulièrement nette dans le domaine de l'éducation (un système éducatif public laïque et aucun financement direct des écoles confessionnelles) ${ }^{11}$.

D'autres associaient explicitement la notion de "secularism " et l'immoralité ou l'amoralité censée régner dans les autres pays européens occidentaux, comme le sénateur Fianna Fáil Don Lydon en 1988 (en réaction à la " Nouvelle Constitution " proposée par les Progressive Democrats à l'occasion du $50^{\mathrm{e}}$ anniversaire de la Constitution irlandaise) :

The Progressive Democrats request that the State be neutral in its attitude to religion. However, by so doing the State would be identifying itself with secularism and acknowledging, wrongly, that religion has nothing to do with the common good. The unbounded pluralism espoused by the Progressive Democrats and others would contribute to the loss of the sense of God and to the increasing depersonalisation that we witness today in many western European societies ${ }^{12}$.

On retrouve le sens proche d' " anti-religieux " dans les discours politiques d'un Bertie Ahern (alors Premier ministre) fustigeant les "aggressive secularists " (jamais nommés au demeurant) qui chercheraient à faire disparaître la religion du domaine public et donc par amalgame de la société elle-même ${ }^{13}$. Devant une telle stigmatisation du terme, il n'est pas étonnant que la plupart des politiciens de premier plan se défendent de faire partie de ces "sécularistes ", comme cela a été le cas du travailliste irlandais Ruairí Quinn, nouvellement nommé ministre de l'Éducation au printemps 2011, au moment de l'annonce du Forum "Pluralism and Patronage ${ }^{14}$ ".

Lorsque "secular " n'est pas utilisé dans ce sens, il est parfois employé de telle façon que la formulation porte à confusion, comme dans une phrase prononcée par Bertie Ahern au retour de sa visite du pape en 2005 :

Our future can only be shaped with confidence when we are secularly rooted in our culture and in our community. One pillar of that culture is the Christian tradition. It is a culture of community, but one with deep respect for the individual ${ }^{15}$.

10. Patrick Cooney, 16 juin 1987, Dáil Eireann Debate, vol. 373, n 8.

11. Jean Baubérot, Les Laïcités dans le monde, Paris, PUF, 2009, p. 87; Élizabeth Zoller (dir.), La Conception américaine de la laïcité, Paris, Dalloz, 2005.

12. Don Lydon, 11 fév. 1988, Seanad Eireann Debate, vol. 118, nº 10.

13. Cité dans Patsy McGarry, "Ahern 'upset' by hesitation to publicly debate faith ", Irish Times, 23 avril 2008.

14. Irish Times Reporters, "Quinn defends patronage changes ", Irish Times, 14 avril 2012.

15. Cité dans Jean-Christophe Penet, "Closer to Brussels than to Rome? The EU as the new external referent for a secularised Irish society and a redefined Catholic identity ", Etudes Irlandaises, n 41.1, printemps 2009, p. 65. 
Cette idée d'un enracinement " laïque » dans une culture dont une caractéristique fondamentale serait la tradition chrétienne paraît typique d'une vision commune à nombre de députés Fianna Fáil en particulier, le prémisse de base étant la possibilité d'afficher un soutien public à la fois à la séparation de l'Église et de l'État et à la tradition chrétienne, une tendance que l'on retrouvera également chez des députés du Fine Gael, et un mélange des genres pour le moins douteux dans une perspective de neutralité de l'État vis-à-vis des religions.

Jusqu'aux années 2000, la tendance dominante des milieux politiques irlandais restait ainsi d'associer la séparation de l'Église et de l'État purement et simplement avec l'absence d'une religion d'État reconnue officiellement - avec l'idée que la question avait été réglée avec l'amendement constitutionnel de 1972, alors que les spécialistes s'accordent sur le fait qu'une telle absence ne suffit pas à elle seule à établir cette séparation dans les faits ou autrement dit à faire de l'État un État laïque, neutre vis-à-vis des religions. On en revient donc à la question du sens des mots "séparation " et " neutralité " dans le discours politique irlandais appliqué à différents domaines de la vie politique et sociale en Irlande.

\section{Une conception idiosyncratique et problématique de la neutralité de l'État en matière de religion}

La conception de la neutralité de l'État prônée plus ou moins implicitement dans les milieux politiques irlandais au moins jusquaux années 1960 revenait en réalité à une attitude de "non-ingérence " légitimant la position de donneur d'ordre de l'Église catholique en matière à la fois sociale et éducative. L'État " neutre » se devait de ne pas intervenir dans les affaires de l'Église, et les affaires de l'Église étaient comprises comme incluant entre autres le contrôle de l'éducation (car figurant parmi ses missions primordiales) - avec malgré tout une implication forte de l'État dans la définition des programmes hors instruction religieuse comme dans le financement des écoles. La hiérarchie administrative publique se subordonnait de fait à la hiérarchie administrative religieuse dans un certain nombre de domaines ${ }^{16}$, la légitimité et la primauté de la mission éducative religieuse étant acceptées dans ces écoles "nationales ", une position renforcée par le présupposé que les Églises pouvaient légitimement agir au nom des parents, «éducateurs premiers des enfants " selon la Constitution. Avec l'exception notable de la réforme de l'enseignement secondaire par Donagh O'Malley (Fianna Fáil) en 1967 menée à bien malgré l'opposition de l'Église, on peut parler d'une politique consensuelle en la matière ${ }^{17}$.

16. Séamas Ó Buachalla, Education Policy in Twentieth Century Ireland, Dublin, Wolfhound Press, 1988, p. 250-278. 17. Ibid. 
Le contexte discursif de nombre des allusions faites à la séparation de l'Église et de l'État dans les débats parlementaires des années 1970-1980 montre que, pour les députés en question, celle-ci ne renvoyait toujours pas à la notion de neutralité de l'État vis-à-vis des Églises et des croyances mais bien à l'idée que l'État ne devrait pas interférer dans les affaires de l'Église ou même parfois que des représentants de l'État devraient s'abstenir de tout commentaire concernant l'Église. L'interprétation de "non-ingérence » semble la seule qui puisse permettre de comprendre des déclarations comme celle du sénateur Fianna Fáil Don Lydon, lorsqu'il affirmait en 1988, dans un discours de défense de la "république chrétienne " : "I certainly support the separation of Church and State. [...] Ireland is primarily a Christian society guided by Christian principles and such principles need the support of law ${ }^{18}$."

Entre les années 1980 et 2000, on trouve plusieurs illustrations de la tendance politique forte assimilant neutralité et non-ingérence dans les débats parlementaires, suite à des questions généralement posées par des parlementaires travaillistes, avec des ministres de l'Éducation refusant expressément d'envisager une quelconque intervention de l'État d'un point de vue institutionnel et choisissant de présenter ces aspects structurels comme ne relevant pas du rôle de l'État, ou à tout le moins de se restreindre à une présentation de la pratique existante. En 1985, Gemma Hussey, ministre de l'Éducation (Fine Gael), choisit cette ligne, en réponse à Michael $\mathrm{D}$. Higgins qui soulevait le problème de la discrimination religieuse pour les enseignants (et ce malgré le fait qu'elle avait elle-même à l'époque envisagé la mise en place d'autorités locales en matière d'éducation, projet vite avorté $^{19}$. On peut rapprocher ce positionnement de celui de Mary Hanafin, ministre de l'Éducation Fianna Fáil au milieu des années 2000 : en réponse à des journalistes qui l'interrogeaient dans le contexte de la controverse de Balbriggan en 2007 (enfants de parents immigrés, dont de nombreux Africains, refusés dans l'école catholique locale), Mary Hanafin avait insisté sur le fait qu'il n'y aurait aucun changement législatif, dans la mesure où la législation " reflète le système éducatif ${ }^{20}$ ". Un an plus tôt, en réponse à des commentaires faits cette fois encore par un parlementaire travailliste sur la nécessité de changements structurels dans l'éducation, Mary Hanafin avait exprimé très clairement la primauté institutionnelle des Églises dans le système éducatif irlandais, dans une déclaration d'impuissance auto-proclamée de l'autorité publique :

18. Don Lydon, 11 février 1988, Seanad Éireann Debate, vol. 118, n 10. Membre du Seanad de 1987 à 2007.

19. Gemma Hussey, 11 décembre 1985, Seanad Eireann Debate, vol. 110, n 7.

20. Karin Fischer, «Adapting the School System to the Globalization of Ireland's Population: an Irish Solution to an Irish Problem?", The Irish Review, 40-41, hiver 2009, p. 149-151. 
I have no intention of changing the management of schools throughout the country. I am not the manager or owner of schools. I do not own the sites and I am not a patron, so it is not possible to do $\mathrm{so}^{21}$.

Dans le domaine de l'éducation toujours, on a pu voir s'opérer néanmoins entre les années 1980 et 2000 un glissement dans la conception de la neutralité de l'État, de l'idée de non-ingérence à une position affichée d'État arbitre, voire d'État « facilitateur » de différents intérêts religieux et/ou privés dans l'éducation. Ce glissement reflète le passage du "paradigme théocratique " au "paradigme mercantile " analysé par Denis O'Sullivan ${ }^{22}$. Il renvoie à un nouveau discours politique dominant centré sur le rôle de l'éducation dans l'économie, mais marqué également par l'usage de notions telles que "pluralisme " et "diversité ", véhiculant le message implicite d'une Irlande État démocratique moderne ayant dépassé la dévolution traditionnelle de l'éducation aux Églises chrétiennes comme seuls pourvoyeurs possibles ${ }^{23}$. Une certaine distanciation vis-à-vis des Églises et de la tradition chrétienne était donc visible dans le discours mais on peut malgré tout parler de confusion persistante sur le sens de la notion de neutralité de l'État en matière religieuse. Les gouvernements Fianna Fáil successifs des années 2000 ont ainsi ramené cette notion de neutralité à une attitude officielle d'égalité entre les "patrons" ou fondateurs-propriétaires des écoles, une attitude elle-même sujette à caution et au final impossible à tenir ${ }^{24}$.

Le positionnement de "non-ingérence » ou de partenaire passif resta si marqué de la part des gouvernements Fianna Fáil successifs au cours des années 1990 et 2000 que c'est l'Église catholique elle-même, ou du moins certaines voix en son sein, comme l'évêque de Meath en 2000, qui commencèrent à demander à l'État d'assumer une responsabilité dans la mise à disposition et l'administration d'écoles : «Dr. Smith [Bishop of Meath] took issue with the fact that the Department of Education expected the Catholic Church to provide and run all or most schools ${ }^{25}$." C'est ainsi sur la demande expresse de l'Église catholique que les premières évolutions politiques ont eu lieu dans ce domaine, l'Église elle-même reconnaissant la réalité de la diversification religieuse de la société irlandaise et suggérant une diversification correspondante des "patrons »/propriétaires-fondateurs d'écoles ${ }^{26}$.

21. Mary Hanafin, 15 fév. 2006, Dáil Éireann Debate, vol. 614, n 45.

22. Denis O'Sullivan, Cultural Politics and Irish Education since the 1950s - Policy, Paradigms and Power, Dublin, Institute of Public Administration, 2005.

23. Ibid.

24. Eoin Daly, "Religious Freedom as a Function of Power Relations: Dubious Claims on Pluralism in the Denominational Schools Debate ", Irish Educational Studies, vol. 28, 2009, p. 235-248.

25. Sean Flynn, "Bishop queries non-believers' availing of Catholic schools ", Irish Times, 19 mai 2000.

26. Cf discours de Diarmuid Martin, archevêque de Dublin et Primat d'Irlande, au colloque organisé par le ministre de l'Éducation le 27 juin 2008 sur le thème " Governance Challenge for Future Primary Schools ", [http://www.dublindiocese.ie/content/27062008-governance-challange-future-primary-school-needs-kilmainham] (au 20 août 2014). 
L'essentiel du débat politique irlandais jusqu'à présent (gouvernement Fine GaelLabour depuis 2011 inclus) est ainsi resté dans les rails suggérés par la hiérarchie catholique et dans l'esprit de ses propositions ${ }^{27}$.

La proposition de diversification peut être considérée comme une stratégie logique pour l'Église catholique, qui voit l'équation pluralisme/diversité d'écoles comme un moindre mal, une façon d'éviter précisément toute solution systémique de type laïque, et donc de conserver le plus grand nombre possible d'écoles catholiques. Une telle politique de diversification est nettement plus problématique pour l'État du point de vue du respect de la neutralité en matière de religion. Quelle que soit la conception dominante, attitude de "non-ingérence " ou d'arbitre, les principaux partis irlandais ont jusqu'à présent dévoyé la notion de neutralité de l'État, plus ou moins consciemment ou délibérément peut-être, pour légitimer le modèle irlandais du "patronage ", structure dévolutive qui avait au départ pour but de préserver le rôle des Églises dans le contrôle des écoles, et qui aujourd'hui conduit plus largement à légitimer le contrôle des écoles par des intérêts privés organisés, les propriétaires-fondateurs ayant la main à la fois sur la gestion et sur l'esprit caractéristique spécifique censé animé " leurs " écoles. Une telle vision du pluralisme dans l'éducation est associée à la conception de droits collectifs majoritaires ou minoritaires, sans référence aux droits fondamentaux individuels ou aux implications concrètes que devrait avoir la séparation des Églises et de l'État ${ }^{28}$.

\section{圈 Évitement, contournement, confrontation : réponses politiques diverses et mentalité dominante}

D’une manière générale cependant, on peut parler dans ce domaine d'une stratégie d'évitement plutôt que de légitimation explicite : comme Enda McDonagh l'a fait remarquer, des années 1970 à 1990, les débats sur les relations Église-État portaient quasi exclusivement sur le rapport entre loi et moralité sexuelle, et spécifiquement sur la contraception, le divorce ${ }^{29}$, l'avortement et l'homosexualitée

27. Chapitre " Pluralising the Patronage Model : The Limited Horizons of Reform ", in Daly, Religion, Law and the Irish State, p. 284 et suivantes.

28. Alison Mawhinney, Freedom of Religion and Schools: The Case of Ireland-A failure to protect international human rights standards, Saarbrücken, VDM Verlag, 2009, p. 121, 189-190.

29. Ruairí Quinn, alors ministre du Travail, déclarait, lors d'un débat sur la légalisation du divorce en 1986 : "There is an absolute necessity to make a reality, in perception terms as distinct from legal terms, of the separation of Church and State in this country [...]. We need to make the dream of an Irish Republic, which sustained so many generations of unfree Irishmen and women, a reality before the end of this century ». 26 fév. 1986, Dáil Éireann Debate, vol. 364, n 2.

30. Enda McDonagh, "Church-State Relations in an Independent Ireland ", in James P. Mackey et Enda McDonagh (dir.), Religion and Politics in Ireland at the turn of the millennium, Dublin, Columba Press, 2003, p. 53 . 
(en dehors du débat sporadique sur le problème de la fermeture des pubs le Vendredi Saint en cas de match de football) ${ }^{31}$. L'accent mis sur ces aspects polémiques tendait à éclipser d'autres questions de liberté individuelle et de droits fondamentaux, y compris dans le domaine social ou de la santé. Les débats politiques sur la relation Église-État dans l'éducation et sur ses conséquences en termes d'égalité citoyenne et de liberté de conscience et de religion sont restés rares par comparaison, en dehors d'interventions régulières, mais isolées, de la part de députés ou sénateurs bien connus tels que Michael D. Higgins ${ }^{32}$, Proinsias de Rossa (Democratic Left) et David Norris ${ }^{33}$. Ayant étudié l'évolution des débats politiques et de la société irlandaise sur ces questions de moralité sexuelle, Chrystel Hug avait pu affirmer en 1999 que la société irlandaise était passée d'un ordre moral fondé sur la loi naturelle et sur une idéologie victorienne à un ordre moral fondé sur les droits fondamentaux des individus, et que l'Irlande s'était ainsi éloignée d'une conception primitive de la démocratie fondée sur la loi de la majoritée ${ }^{34}$. Même s'il y a bien eu une certaine évolution, un tel mouvement politique est beaucoup plus difficilement perceptible dans le domaine de l'éducation.

C'est par le biais des débats politiques suite aux révélations des Rapports Ryan (2009), Murphy (2009) et Cloyne (2011) sur les mauvais traitements, dont des agressions sexuelles, infligés aux enfants par des prêtres ou membres d'ordres religieux (notamment au sein d'écoles sous le contrôle de l'Église) que la question du rapport État-Église a ressurgi, soulevant cette fois également la question du rôle de l'État dans l'éducation, quoique de manière toujours très périphérique dans les débats. Les sénateurs indépendants Joe O’Toole ${ }^{35}$ et David Norris ont ainsi été parmi les très rares parlementaires à lier explicitement la question du rapport entre Église et État soulevée par ces Rapports à celle de la place des Églises dans le système éducatif irlandais. David Norris déclarait en décembre 2009 :

31. Joe O’Toole, 11 mai 2000, Seanad Éireann Debate, vol. 163, n 5; 9 mars 2010, Seanad Éireann Debate, vol. $201, \mathrm{n}^{\circ} 7$.

32. Parmi ses nombreuses interventions sur le sujet : Michael D. Higgins, 11 déc. 1985, Seanad Eireann Debate, vol. $110, n^{\circ} 7 ; 16$ juin 1987, Dáil Eireann Debate, vol. $373, n^{\circ} 8$.

33. David Norris (lui-même membre de l'Église d'Irlande), au sujet d'une nouvelle disposition législative prise en 2000 qui légalisait une forme pré-existante de discrimination religieuse en permettant explicitement au centre de formation des enseignants protestant de réserver l'inscription à des étudiants protestants : « In a Republic we should try to ensure that all citizens are treated equally. Let us openly acknowledge that this is a variation on this ». La disposition législative fut adoptée au nom du " droit des minorités » et dans un consensus apparent, en dehors donc de la remarque isolée de Norris. David Norris, Employment Equality Act, 1998 (Section 12) (Church of Ireland College of Education) Order, 2000, 29 juin 2000, Seanad Éreann Debate, vol. 163, nº 25. David Norris, 10 oct. 2007, Seanad Éireann Debate, vol. 187, n 6.

34. Chrystel Hug, The Politics of Sexual Morality in Ireland, Houndmills, Basingstoke (UK), Macmillan Press, 1999, p. 241-242.

35. Joe O’Toole : élu de la circonscription de la National University of Ireland au Seanad, ancien Président de l'Irish Congress of Trade Unions et ancien Secrétaire Général de l'Irish National Teachers' Organisation. 
It is all very well for [the Minister for Justice, Equality and Law Reform] to say that no Church can be above the law, but the Churches are above the law. This House placed them above the law in the particularly sensitive area of children in education. We gave them exemptions from the operation of equality legislation, something no Christian Church in conscience should ever have asked for. It was a disgrace at the time and only Senator Joe O'Toole and myself opposed it in this House, which is also reprehensible ${ }^{36}$.

Est apparu néanmoins un questionnement sur la réalité de la séparation Église/ État en Irlande de la part de membres de partis politiques qui jusque-là avaient généralement préféré considérer celle-ci comme acquise, comme le Fine Gael avec l'exception remarquable de Garret FitzGerald, chef du parti de 1977 à 1987 et Premier ministre en 1981-1982 et 1982-198737. Lors du débat parlementaire lié à la publication du Rapport de la Commission d'investigation sur le diocèse catholique de Cloyne en juillet 2011 (enquête sur les responsabilités respectives de l'Église catholique et de l'autorité publique dans une série de cas de violences sexuelles sur des enfants par des prêtres) ${ }^{38}$, la députée Fine Gael Michelle Mulherin présentait la séparation Église-État comme impérative. Elle ne sous-tendait pas moins cet impératif d'une argumentation très explicitement chrétienne, à laquelle elle semblait associer l'ensemble du Dáil, voire la population irlandaise, par l'emploi d'un « nous, chrétiens " globalisant :

The separation of Church and State is an imperative. It is in keeping with the worldly authority given to us as Christians. If it were not the case that the rule of the Earth was given to men and women, surely the perpetrators of these most heinous crimes, including some priests, would be struck down by God. We know this has not happened. Therefore, it is a matter for us as human beings to correct these wrongs politically and by means of the law. In this case, we can do so by introducing appropriate legislation. This domain is our God-given responsibility ${ }^{39}$.

36. David Norris, $1^{\text {er }}$ déc. 2009, Seanad Éireann Debate, vol. 198, $\mathrm{n}^{\circ} 9$.

37. Au début des années 1980, Garret FitzGerald, alors Premier Ministre, s’était retrouvé isolé dans son propre parti lorsqu'il avait déclaré que l'État restait marqué par un « esprit majoritaire " (d'un point de vue religieux - « majority ethos ») et qu'il souhaitait lancer une " croisade républicaine pour la création d'une république authentique » (" a republican crusade to make this a genuine republic "). Enda McDonagh, "Church-State Relations in an Independent Ireland ", in James P. Mackey and Enda McDonagh (dirs), Religion and Politics in Ireland at the turn of the millennium, Dublin, Columba Press, 2003, p. 51.Voir aussi Barre Fitzpatrick et Garret FitzGerald, "The Politics of Pluralism : Interview with Garret FitzGerald ", The Crane Bag, vol. 5, n 1, " Minorities in Ireland " (1981), p. 50 ; Dermot Keogh, "Catholicism and the Formation of Modern Irish society », in Princess Grace Irish Library (dir.), Irishness in a Changing Society, Monaco, Princess Grace Irish Library, 1988, p. 173.

38. Report by Commission of Investigation into the handling by Church and State authorities of allegations and suspicions of child sexual abuse against clerics of the Catholic Diocese of Cloyne, [justice.ie/en/JELR/Pages/ Cloyne_Rpt.]

39. Michelle Mulherin, 20 juillet 2011, Dáil Éireann Debate, vol. 739, n 3 . 
Dans le même contexte, le chef du gouvernement lui-même, Enda Kenny (Fine Gael), a tenu à mettre les points sur les i concernant la distinction entre loi de l'État et loi canon (et donc à admettre implicitement que cette distinction n'allait toujours pas de soi en Irlande) dans un discours très remarqué le 20 juillet 2011, discours qui a été interprété comme une déclaration d'indépendance morale et législative de l'État irlandais vis-à-vis du Vatican ("This is the "Republic" of Ireland 2011 ") $^{40}$.

Enda Kenny est cependant resté soucieux de circonscrire la question au domaine de la protection des enfants contre les mauvais traitements ou abus sexuels, une question qui a abouti finalement à un amendement constitutionnel sur les droits des enfants adopté par référendum en 2012 (suspendu jusqu’à la décision de la Cour suprême suite à un recours juridique). Si le discours insistait sur l'indépendance législative de l'État irlandais et sur sa qualité de république démocratique, l'emploi d'un certain vocabulaire renvoyait néanmoins davantage à une vision religieuse chrétienne qu'à une conception fondée sur le droit : Enda Kenny déclarait que l'Église devait être responsable devant la loi, mais aussi qu'il fallait qu'elle soit une "Église pénitente ". Surtout, plutôt que de mettre l'accent sur la notion de droits de l'enfant, Kenny choisissait d'évoquer le devoir de "protéger l'espace sacré de l'enfance et de restaurer son innocence $e^{41}$ ».

Malgré l'évolution relative que semblait annoncer ce discours d'Enda Kenny, un désaccord de fond, mais aussi une certaine confusion, semble bien avoir persisté au sein de la classe politique sur la réalité concrète ou non de la séparation entre l'Église et l'État en Irlande. Comme d'autres analyses historiques le confirment ${ }^{42}$, il est clair que la majorité des députés du Fianna Fáil quant à eux continuaient à partager au fond la vision d'une "république chrétienne ", une conception qui permettait au final un rejet de l'idée même de neutralité, et donc entre autres de toute véritable remise en cause de la place des Églises dans le système éducatif irlandais ${ }^{43}$.

Les principaux partis ont donc continué à refuser de s'attaquer de front à la question, et, des années 1970 à 2011, les tentatives de porter le débat de la neutralité de l'État sur la place publique irlandaise sont essentiellement restées le fait de politiciens isolés, le plus souvent sans appui officiel explicite de leur parti sur le sujet. Cela a été le cas historiquement au sein du Parti travailliste : on trouve

40. Statement on the Dáil Motion on the Report of the Commission of Investigation into the Catholic Diocese of Cloyne, in Dáil Éireann, by Taoiseach Enda Kenny, 20 juillet 2011.

41. " to protect the sacred space of childhood and to restore its innocence " (mes italiques). Enda Kenny, 20 juillet 2011, Dáil Éireann Debate, vol. 739, n³.

42. Keogh, "Catholicism and the Formation of Modern Irish society ", p. 152-177.

43. Voir par exemple les interventions de députées Fianna Fáil pour une diversification limitée des écoles et pour la préservation des écoles catholiques "dans nos paroisses ", 20 mai 2010, Dáil Eireann Debate, vol. 709, $n^{\circ}$ 4.Parmi les rares exceptions, Pat Carey, député Fianna Fáil (1997-2011), cité dans Patsy McGarry et Harry

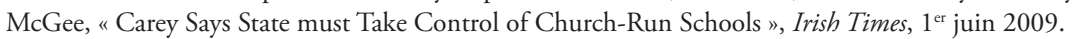


dans les débats parlementaires de nombreuses prises de position individuelles de membres du parti comme Michael D. Higgins au cours des dernières décennies, Pat Rabbitte, notamment lorsqu'il était chef du parti entre 2002 et 2007, ou encore Ivana Bacik ${ }^{44}$, sénatrice travailliste depuis 2007 , mais on ne peut pas pour autant parler de positionnement collectif cohérent sur le sujet de la part du Parti travailliste irlandais en tant que tel, signe de débats persistants sur le sujet en son sein, et peut-être là aussi d'une stratégie d'évitement d'un sujet toujours brûlant en Irlande.

Un nombre croissant de parlementaires, essentiellement au sein du Parti travailliste ou issus des rangs de la gauche radicale (voir plus loin), n'en appellent pas moins de leurs vœux une séparation complète de l'Église et de l'État en Irlande, en faisant généralement appel aux fondements républicains de l'État, et vont jusqu'à envisager les conséquences qu'une telle séparation devrait avoir dans l'éducation. C'est également le cas de députés du Sinn Féin tel qu’Aengus Ó Snodaigh ou Arthur Morgan notamment (ces derniers insistant spécifiquement sur le déficit démocratique du système éducatif irlandais, à l'exception des écoles des VEC) $)^{45}$.

Le Sinn Féin, comme le Parti travailliste, reste malgré tout très prudent dans ses déclarations officielles en dehors du Dáil ${ }^{46}$. Les Verts paraissent eux aussi ouverts à des évolutions dans le sens d'une séparation plus nette, mais là encore sans prise de position globalisante sur le sujet. Parmi les députés du Parti vert, tandis que Ciaran Cuffe en 2003 revenait sur la nécessité de reformuler le Préambule et les articles 6, 12, 34 et 44 de la Constitution irlandaise pour rendre effective la séparation de l'Église et de l'État ${ }^{47}$, Paul Gogarty quant à lui ne voyait pas d'inconvénient à ce que les écoles nationales continuent pour la plupart à promouvoir leur esprit catholique ${ }^{48}$. Le respect du " choix des parents " comme priorité politique permet aux uns et aux autres d'encourager une diversification des écoles, et notamment le développement des écoles Educate Together (facilité depuis l'élection législative de 2011), sans pour autant s'attaquer directement à la question toujours manifestement trop épineuse du respect de la neutralité de l'État en matière de religion.

De fait, jusqu’à présent, seuls de petits partis, pas toujours représentés au Dáil, ont adopté une politique explicite de séparation complète de l'Église et de l'État et d'élimination de toute discrimination religieuse dans le domaine éducatif (comme dans les autres domaines) en République d'Irlande. Il s'agit d'un côté des Progres-

44. Ivana Bacik, 10 oct. 2007, Seanad Éreann Debates, vol. 187, nº 6.

45. Aengus Ó Snodaigh, 20 mai 2010, Dáil Eireann Debate, vol. 709, n 4; Arthur Morgan, 13 oct. 2010, Dáil Éreann Debate, vol. $718, \mathrm{n}^{\circ} 2$.

46. Sinn Féin, Education and Childcare : Reaching our Full Potential - Sinn Féin Education and Childcare Platform 2007, p. 2.

47. Ciaran Cuffe, 25 nov. 2003, Dáil Éireann Debate, vol. 575, nº 3.

48. Paul Gogarty, 13 oct. 2010, Dáil Éireann Debate, vol. 718, nº 2. 
sive Democrats (parti néolibéral et conservateur, tenant d'un certain libéralisme social et politique, créé en 1985 et qui a cessé d'exister en 2009), avec leur Constitution pour la Nouvelle République à la fin des années $1980^{49}$ (en contradiction avec leur allié politique, le Fianna Fáil), et de l'autre des petites formations de la gauche dite radicale, en particulier le Workers' Party et le Parti socialiste irlandais (Joe Higgins) ${ }^{50}$ sur une base démocratique républicaine de respect des droits fondamentaux individuels. En témoignent notamment les positions adoptées par les députées Clare Daly (Socialist Party) et Joan Collins (People before Profit Alliance) lors du débat de juillet 2011. Voix nouvelles dans le paysage parlementaire irlandais ${ }^{51}$, ces dernières appelaient sans détours à une séparation de l'Église et de l'État dans une perspective laïque et fondée sur l'exigence du respect des droits fondamentaux de la personne ${ }^{52}$. Clare Daly revenait aussi sur le caractère problématique de la prière marquant chaque jour le début des travaux du Dáil, une question régulièrement soulevée auparavant par David Norris en particulier ${ }^{53}$.

Le présent article portait sur la période des années 1970 à 2011. Il faut signaler que ces dernières années ont vu une approche à la fois plus offensive et plus largement partagée de certaines questions liées à la séparation de l'Église et de l'État, comme la persistance de formes de discrimination religieuse légales au sein des établissements scolaires ou de santé gérés par les Églises, permises par la fameuse clause 37 de la loi sur l'égalité dans l'emploi de 1998. Le débat au Seanad autour de la proposition d'amendement de cette loi avancée par la sénatrice travailliste Ivana Bacik en 2013, avec des déclarations de soutien de membres du Fine Gael et du Fianna Fáil en particulier ${ }^{54}$, s'est en effet déroulé dans une atmosphère consensuelle très différente de celle qui avait présidé à l'adoption de cette clause en 1998. Comme Kathleen Lynch ${ }^{55}$ (députée travailliste et Secrétaire d'État dans le gouvernement de coalition Fine Gael-Parti travailliste depuis 2011) l'a fait elle-même remarquer au cours du débat de 2013, elle s'était retrouvée au contraire bien isolée dans son opposition à la clause d'exemption lors des débats sur le sujet en $1996^{56}$.

L'étude de l'emploi, somme toute relativement rare, du terme "secular " (et de son dérivé plus souvent utilisé "secularist») dans les débats parlementaires irlan-

49. Progressive Democrats, Constitution for a New Republic, janvier 1988.

50. Par exemple Joe Higgins, 13 déc. 2005, Dáil Éireann Debate, vol. 612, n 105. Ces deux partis, comme l'organisation People before Profit, étaient membres de la United Left Alliance, alliance électorale créée à l'occasion des élections de 2011.

51. Toutes deux élues au Dáil en 2011 sous la bannière de la United Left Alliance, Clare Daly et Joan Collins ont depuis fondé ensemble un nouveau parti, United Left (mai 2013).

52. 20 juillet 2011, Dáil Éireann Debate, vol. 739, n 3 .

53. Entre autres David Norris, 21 juillet 2011, Seanad Éireann Debate, vol. 209, nº 12.

54. Employment Equality (Amendment) (n²) Bill 2013: Second Stage, Seanad Debates, 13 mars 2013.

55. Minister of State for Primary Care, Mental Health and Disability, précédemment Minister of State for Disability, Equality and Mental Health.

56. Kathleen Lynch, Seanad Debates, 13 mars 2013. 
dais et dans les journaux sur la période des années 1970 à 2000 fait ressortir une impression de confusion sémantique, ou à tout le moins une acception réductrice voire tendancieuse du terme. Son rejet dans le monde politique irlandais à quelques exceptions près paraît révélateur de cette confusion et de cette ambiguïté persistantes, et finalement du fait que la République d'Irlande ne peut pas prétendre avoir atteint une forme complète ou même avancée de séparation de l'Église et de l'État, malgré les déclarations en ce sens.

L'étude des discours politiques autour de la notion de neutralité de l'État fait ressortir une conception politique dominante qui a évolué avec le temps, reflétant pour une bonne part le passage du paradigme théocratique au paradigme mercantile analysé par Denis O'Sullivan dans son ouvrage sur l'évolution de la politique éducative en République d'Irlande. La conception irlandaise de la neutralité de l'État en matière religieuse dans l'éducation a ainsi évolué d'une attitude de "noningérence » à un positionnement affiché d'arbitre entre différents intérêts religieux et/ou privés. Plus récemment (et surtout depuis 2011 et l'arrivée au pouvoir de la coalition Fine Gael-Parti travailliste), l'État, par l'intermédiaire du ministre de l'Éducation Ruairí Quinn, s'est présenté comme étant au service du choix des parents $s^{57}$, au sein d'un système resté à ce jour largement identique, et avec toutes les difficultés qu'une telle approche peut soulever ${ }^{58}$ - un nouveau positionnement qui préfere toujours éviter ou plutôt contourner pour l'essentiel les questions de neutralité ou de séparation Église-État dans ce domaine, même si Ruairi Quinn s'est voulu plus attentif aux droits des personnes concernées et à la question de la liberté de conscience et de religion ${ }^{59}$, notamment suite aux résultats d'une enquête menée par la Irish Human Rights Commission en 2011 d'une part ${ }^{60}$, et à la publication du rapport lié au "Forum on Patronage and Pluralism " dont il avait été à l'initiative en 2011-2012 d'autre part ${ }^{61}$.

En dépit de cette évolution récente, l'analyse permet aussi de confirmer, et peut-être de mieux comprendre, la persistance de ce que Bill Kissane a décrit comme «l'illusion de la neutralité de l'État ». Il est frappant de constater de fortes similitudes à la fois dans les discours ou écrits dits conservateurs (en faveur d'une forme de statu quo structurel dans l'éducation) et dans ceux qui véhiculent une

57. Communiqué de presse du ministère de l'Éducation, " Minister Quinn publishes the report of the Advisory Group to the Forum on Patronage and Pluralism in the Primary Sector ", 10 avril 2012.

58. Daly, Religion, Law and the Irish State, p. 324-328.

59. Communiqué de presse du ministère de l'Éducation, « Update on Forum on Patronage and Pluralism in Primary Sector published by Minister Quinn ", $1^{\text {er }}$ juillet 2014, et publication correspondante, [http://www.education.iel en/Press-Events/Conferences/Patronage-and-Pluralism-in-the-Primary-Sector/Progress-to-Date-and-Future-Directions-

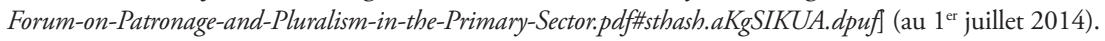

60. Irish Human Rights Commission, Religion and Education: A Human Rights Perspective, Dublin, IHRC, mai 2011.

61. Report of the Advisory Group to the Forum on Patronage and Pluralism in the Primary Sector, Dublin, Department of Education and Skills, avril 2012. 
position plus ouverte à des changements importants. D'où l'idée d'une " mentalité politique "spécifique, au sens utilisé par George Lakoff dans The Political Mind (Lakoff, chercheur en sciences cognitives, s'est intéressé à l'étude des cadres de référence sémantiques dans le domaine politique) ${ }^{62}$, même s'il existe malgré tout, particulièrement au sein du Parti travailliste mais aussi dans les autres partis, quoique de manière encore plus isolée peut-être (si on choisit de n'évoquer que les partis traditionnellement présents au Dáil), un certain nombre de personnalités ayant développé et adopté un positionnement net en faveur d'une séparation complète de l'Église de l'État et de l'élimination concomitante de toute forme de discrimination religieuse. Plus largement, le débat actuel sur la nature républicaine de l'État irlandais et les appels à une "république réelle » ou une «seconde république » ont amené divers commentateurs à s'attaquer directement à la question des caractéristiques fondatrices d'une république démocratique, et contribué à relancer, parmi d'autres, le serpent de mer de la séparation de l'Église et de l'État en Irlande ${ }^{63}$.

62. George Lakoff, The Political Mind, New York, Penguin Books, 2009.

63. Entre autres Fintan O’Toole, Enough is Enough - How to Build a New Republic, Londres, Faber and Faber, 2010. 